\title{
Discourse Analysis Techniques for Modeling Group Interaction *
}

\author{
Alexander Feinman and Richard Alterman \\ Computer Science Department, Brandeis University MS018, \\ 415 South St., Waltham MA 02454 \\ [afeinman, alterman] @cs.brandeis.edu
}

\begin{abstract}
This paper presents discourse analysis techniques that model the interaction of a small group of users engaged in same-place/differenttime interaction. We analyzed data from VesselWorld, our experimental testbed, and formulated a modeling technique based on the recurrence of coordination problems and the structure that users create to handle these problems. Subsequent experiments revealed that our original analysis had failed to capture issues with the cognitive load required to maintain common ground. By tracking references users make to both domain and conversational objects, we were able to extract patterns of information access and model the cognitive load incurred to maintain common ground. The improved model of user interaction was successful in explaining systems designed to support interaction.
\end{abstract}

\section{Introduction}

Groupware applications for supporting interaction can be very hard to use, sometimes impairing the work they are built to support. Design of successful groupware applications is challenging, for reasons ranging from technical concerns to sociological issues of introducing groupware into an existing interaction. Ideally, a software system is built to support the emergent practice of work for a community of users. For groupware systems, this requires modeling the interaction of users as they perform their work.

This paper presents discourse analysis techniques that model group interaction, and analyzes results based on data collected using our groupware testbed, VesselWorld [9]. Initially, we analyzed experimental data from VesselWorld to model recurrent issues of coordination and investigate the conversational structure that users created. This analysis indicated that we needed to model the cognitive load of maintaining common ground. By building a model of how users publish, access, and modify information, we can determine how a system should mediate that information to improve performance. In particular, the length of time that a topic is relevant, the frequency of reference to the topic over that time, and the pattern of access to the information all serve as useful indicators of

\footnotetext{
* This research was supported by the Office of Naval Research under grants No. N00014-96-1-0440 and N66001-00-1-8965
} 
cognitive load and indicate how to present and store information. This method of modeling user interaction explains observed results from the VesselWorld experimental data, and can be used to create systems that properly support the way users exchange information or adapt existing systems to improve such support.

\section{Modeling Recurrence in Coordination}

To study issues in same-time/different-place coordination [5], our lab constructed the VesselWorld groupware system. Three users conduct a simulated clean up of a harbor via a graphical interface. The harbor contains toxic waste that must be cleared safely to a large garbage barge. As the users interact, the system logs all actions and communication for later analysis. This makes VesselWorld ideal for exploring issues of group interaction. At this time, we have collected over 250 hours of data from 18 groups using various versions of VesselWorld.

Each of the three users acts as the captain of a ship navigating the harbor: two users pilot ships with waste-retrieval cranes attached (referred to as crane1 and crane2), allowing them to lift and load barrels of toxic waste; the other user pilots a tugboat (referred to as tug1), and is able to move small barges around the harbor, identify waste, and seal the leaks caused by mishandling of waste. The harbor is cleared in a turn-based fashion, with each user explicitly planning actions for each turn before submitting them to the system for evaluation. During a session, the users are physically separated, but are able to communicate freely via a textual chat facility built into the VesselWorld system.

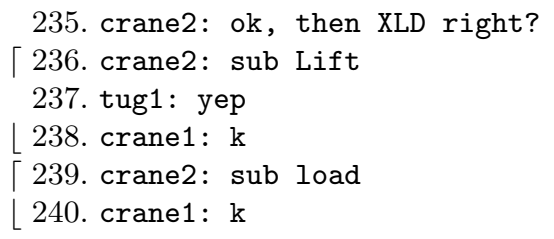

Fig. 1. Adjacency pairs in dialogue from the VesselWorld system.

Despite the simplicity of the harbor-clearing task, users of the VesselWorld system had extensive problems staying coordinated. Problems with maintaining common ground, communicating intentions, and managing information appear throughout the data. To handle these issues, users created conversational structure. For example, to lift and load a large barrel of waste, the two cranes must submit a lift and load commands in synchronization. This led the users to create discourse structure, such as adjacency pairs seen in Figure 1, to help them stay coordinated.

We turned to discourse analysis to help make sense of the dialog. We made use of theories about rules governing conversational flow [10] and grounding in communication [3] to understand how and why users were constructing conver- 
sational structure. We developed an analytic method that built on this research, searching the discourse for three indicators of areas of potential improvement:

1. Recurring patterns of coordination.

2. Recurring errors in coordination.

3. Development of conversational structure by the users to organize recurrent problems of coordination.

Recurrent patterns of coordination and repeating errors are situations in which the users might introduce conversational structure to better organize activity so as to improve performance. The last indicator goes a step further; in this case, the users have determined a potential area of improvement, and have also devised structure to simplify their conversation about the situation and thereby improve it. Examples of structure generated by users include the invention of notational conventions, like specialized jargon ("XLD" meaning an extra-large waste, dredge equipment required), and conversational procedures that support the coordination of activities, like adjacency pairs.

Of the three indicators, the third seems to be the surest bet for the analyst; the users have added corroborating evidence for a particular analysis of the situation. There are problems, however, with an analysis strategy that relies exclusively on the third indicator. The data would have to be very extensive so the users would have time to generate all the most useful secondary structures. The users might identify a coordination problem they would like to fix, but lack the means to fix the problem; there is some evidence for this in our data. Finally, there is no guarantee that organizational structure users might add would improve the situation at all.

\subsection{Applying the model}

Based on analysis of data generated in our VesselWorld pilot study, three coordinating representations (CRs) - software tools for simplifying context sharing were introduced into VesselWorld: the shared planning window, the object list, and the high-level planning window. These representations were aimed at capturing specific forms of information being passed between the users: fine-grained timing issues in shared planning, coarse-grained planning and ordering issues in high-level planning, and object references in the object list.

One of these, the object list, is pictured in Figure 2. It presents information about barrels of toxic waste in the harbor in a concise tabular format. A user adds an entry to the object list by entering details about it in the entry fields at the top and clicking "Add Entry". The entry then appears in the list of objects, which is visible to all users. Entries can be modified by anyone by clicking on the cells of the table; updates are sent to all users. 


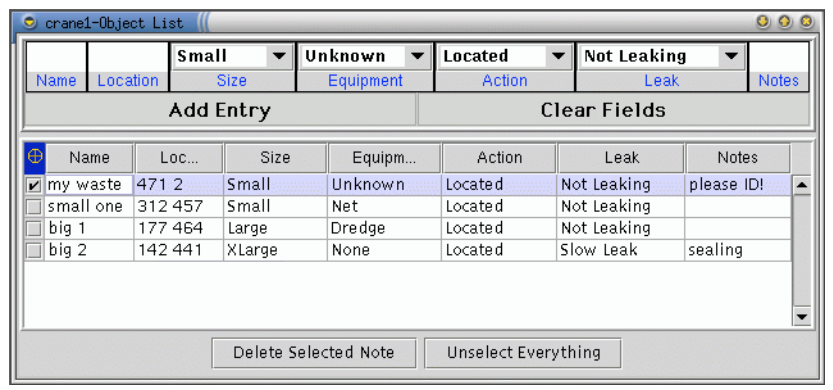

Fig. 2. A coordinating representation: the object list.

\subsection{Results}

To evaluate the coordinating representations we ran the VW3 experiment, completed in early 2001. The experiment was a single-variable study conducted to test the utility of these coordinating representations. Six groups of three people were paid to use VesselWorld for 12 hours each, including training time. We examined only the data from the last five hours for each group, in an effort to avoid interference from the effects of having novice users. Three groups used the base (non-CR) version of Vesselworld, and could only communicate via textual chat. The other three groups used a version that included the three CRs. Differences were dramatic; users of the CR system completed similar problems in $49 \%$ of the clock time that users of the non-CR system required, and committed $61 \%$ fewer errors. A complete summary of experimental results can be found in [1].

Significantly, users did not make full use of the provided CRs. The "Action" column of the object list, dealing with the status of the waste was rarely used, and one of the three CRs, the high-level planning window, was not used at all. To figure out what had gone wrong, we developed an analytic method based on discourse analysis that examined the referential structure of the users' discourse.

\section{Modeling Cognitive Load}

We noted that many of the issues we saw with the existing CRs could be attributed to imbalance in the collaborative effort required to them. For example, using the high-level planning CR seemed to require a lot of effort on the part of the author, while providing only a minimal return on investment. To examine these issues, we created an analysis method centered around the sharing of information between users. In a shared environment like VesselWorld, users interact by negotiating over both domain objects and conversational objects. The initial analysis indicated that tracking these exchanges of information would be useful.

Preliminary analysis of information exchange in the VesselWorld data showed that information of different categories was handled quite differently. Information relating to wastes and other objects in the domain tended to have a long lifetime of relevance (time between first mention and last mention), and the users 
accessed this information infrequently but steadily during that time. Plans, on the other hand, dominated conversation while they were being discussed, but rarely were reused or referred to once agreed upon unless an error occurred. Repairs, where users attempted to fix mismatches in common ground, correct errors in the interaction, or disambiguate misunderstandings, dominated conversation until corrected: only occasionally would another message find its way into the thread of conversation while a repair was underway.

In VesselWorld, information usually has a simple life cycle. Information is reported by a user, either in the chat window, or (in the CR groups) via the object list or shared planning window; it may then be noted by other users; it again becomes relevant at some point and must be retrieved. Information that is relevant over a long span of time may be updated, modified, or otherwise accessed before it is finally rendered irrelevant and forgotten. While information may reside in the short-term memory of the users, storing complex information there can put an unreasonable cognitive burden on the user; distributing the information into the environment [8] yields superior results. However, presenting too much information can lead to information overload. It is important, therefore, to determine what information is worth supporting by constructing CRs. We formed two hypotheses:

1. Information with a long period of relevance is worth recording in a external representation

2. Information which is accessed frequently by the users is worth recording in an external representation

These hypotheses serve as general indicators of when adapting the system may simplify coordination. In the first case, information that is relevant over a long period is very likely to be irrelevant for some subsection of that period. During this time, it represents an unnecessary burden on the user's short-term memory, and in a complex situation with many such items, the burden can easily outstrip the user's ability to memorize. In the second situation, the information is used frequently, and hence needs to be readily available to the users. However, there may be too much information for the user to keep it all in short-term memory. Hence, it needs to be easily and continuously accessible to users as they perform their tasks.

\subsection{Identifying referential structure}

In order to highlight information falling into the above categories, an analyst must track the referents users refer to by thorough examination of the discourse. Past work has looked at reference-tracking to help with the difficult problem of reference resolution ([11]). DeCristofaro et al.'s program REFEREE [4] allowed an analyst to manually tag each reference and attempt to thereby resolve references. Our tool works similarly, with the added functionality of providing tools for statistical analysis of analyst-generated data. 
to the repair, and refers to the already-instantiated iota IOTA-9 by naming it without square brackets. In line 11 , the tug reviews relevant information about the waste. This acts as evidence supporting the repair (that the two references refer to the same waste). The analyst updates IOTA-7's expansion (again using square brackets, this time to indicate that the contents of the iota are being modified), and chooses the earlier of the two names for the waste (IOTA-7 and IOTA-8) to disambiguate further references to the waste.

\subsection{Results}

A great deal of information can be gleaned about group interaction and the passing of information from identifying iotas in the discourse. However, in a long session, it is hard to keep track of the large number of iotas that are generated. To help with the task of interpreting the data we developed a number of special-purpose visualization tools. The access pattern for each iota, that is, when and how it is referred to, can be visualized using a program that extracts the iotas from the analysis, summarizes information about the iotas, and graphically represents the access pattern for each iota as a timeline, greatly aiding interpretation.

\begin{tabular}{l||r|r|r} 
Iota type & Mentions & Lifetime & Density \\
\hline Vessel & 3.1 & 183.6 & $1.7 \%$ \\
Waste & 6.6 & 168.7 & $3.9 \%$ \\
Location & 2.6 & 62.6 & $4.2 \%$ \\
Convention & 5.5 & 109.5 & $5.0 \%$ \\
Barge & 11.9 & 294.0 & $5.6 \%$ \\
Plan & 3.4 & 12.0 & $28.5 \%$ \\
Repair & 3.0 & 4.8 & $62.5 \%$
\end{tabular}

Fig. 4. Selected results from analysis of the VW3 experimental data.

Some results from analyzing the VW3 data are summarized Figure 4. Mentions counts the average number of times an iota is mentioned in any user utterance; for example, in Figure 3, IOTA-8 is mentioned twice. Lifetime is the average number, inclusive, of utterances between first and last mention; for IOTA-8, two. Density is the ratio of these two numbers, and represents the percent of utterances over the life of an iota that refer to that iota; as IOTA- 8 dominates conversation over its short life, its density is $100 \%$. These measures are calculated for each iota, and the average for each type is calculated.

Raw data about lifetime of relevance, frequency of reference, and density of reference can be represented in a scatter plot, to highlight clusters of iotas with similar access patterns. In the semi-log plot shown in Figure 5, the domination of longer lifetimes by waste iotas is clearly visible. Iotas in the upper-left quadrant represent those that by their nature will dominate conversation, and are unlikely 


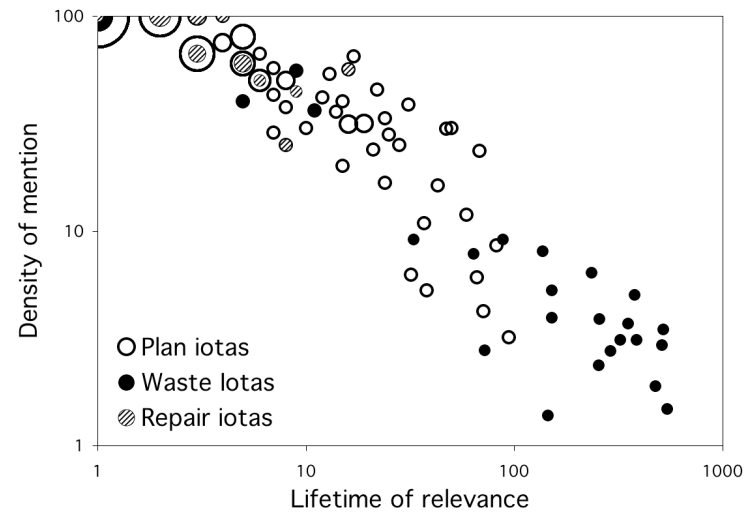

Fig. 5. Distribution of iotas for a non-CR group (larger bubbles indicate multiple iotas)

to require a persistent representation. In contrast, those in the lower-right quadrant of the scatter plot (i.e., wastes) require the most long-term coordination work. From this data we drew conclusions about the workload required to stay coordinated over each sort of information. Here we present results for the most frequent types of iotas used by users in the VW3 experiment, plans and wastes, which together comprised over $80 \%$ of the iotas seen.

Plans As can be seen in Figure 5, plan iotas generally have a short lifetime: $85 \%$ of plans seen lasted 6 or fewer utterances. During this lifetime they had a high frequency of mention, with $50 \%$ or higher density for $70 \%$ of the plans seen. Only in about $10 \%$ of the cases will a plan drag on for some time, generally when it is postponed in favor of another plan; it then shows a pattern resembling two plans strung together, one starting at the initial negotiation, and one starting at the (postponed) re-negotiation.

This distribution of most plans leads us to conclude that, while negotiation of a plan is crucial, their short lifetime means that users will be unlikely to want to put any effort into constructing a shared external representation of their plan. In addition, because a small number of plans co-exist at any one time, and they tend to dominate conversation while they are being discussed, users are probably storing them in short-term memory and using the recent chat for reference. Hence, while an external representation of a plan may serve to ground the plan for multiple users, it is unlikely that users would require persistent storage of plans.

This prediction agrees with the experimental evidence produced by the VW3 experiment. Despite extensive training in its use users refused to use the highlevel planning window, which required significant user effort to create and share a representation of plans, but allowed persistent storage of these plans. The features offered by high-level planning (persistent plan storage, visible plan commitments) were not of sufficient worth to users to outweight the cost of reporting 
their plans via the window. However, they fully embraced the shared planning window, which allowed a low-impact (if less general-purpose) method for sharing near-term intentions. Because it provided good visibility of current intentions, coupled with a low cost to communicate those intentions, shared planning matched the short-term, non-persistent usage pattern of plans.

Shared domain objects The most common type of shared domain object, wastes, have a quite long lifetime of relevance: anywhere from 50 to 500 utterances, depending on session length, complexity, and the order in which the users clear the wastes. In addition, there are short periods of intense activity relating to the wastes, separated by long stretches of up to $40 \%$ of the lifetime where they are not discussed at all. This periodic distribution imposes a heavy load on short term memory for the long stretches where the waste information is not relevant. Also, because of the long lifetime, multiple wastes are often relevant simultaneously; hence, when retrieving information about wastes, users will first need to choose a waste. Storing waste information therefore requires a persistent representation providing easy access and modification capabilities.

In the non-CR system, we provided two methods for discussing wastes: chat history and private markers. As waste is discovered, users generally announce their discovery in chat. Other users could access waste information in the history of their chat, but this was difficult, as it was interleaved with all other communication. Most users used the private markers instead. These allowed a user to annotate his or her map view with a simple note, akin to a sticky note. They were used extensively by most users to keep track of the waste. But markers had a number of problems. First, they effectively tripled the amount of group work needed to stay coordinated, by making each user place a marker in their own private environment. Second, placing a marker required the user to take information from chat, process it correctly, and place a marker in the appropriate position on the map. These complications were a source of great error in non-CR groups. Users often discovered mismatches in their sets of markers, prompting one group to introduce the "marker check", a complex conversational structure, to synchronize these private representations.

The object list, which consolidates information about wastes and other domain objects, addresses these issues. To support the periodic distribution characteristic of waste iotas it is important to construct a persistent and easy-to-access shared representation. The object list gives such a shared, persistent representation. Despite the relatively high cost of formulating an object in the object list (generally 15-30 seconds of interface work, mixed between mouse and keyboard), users willingly performed the task, perhaps sensing a return on the time investment: times for retrieving waste information were as high as a minute when the user had to scan through the chat history, in comparison to a few seconds required to scan of the object list. By providing an authoritative shared source of waste information, mismatches in common ground were reduced. Overall collaborative effort was therefore greatly reduced. 


\section{Future Work}

We are working on quantifying the costs associated with communication for maintaining common ground. Users follow a path of least collaborative effort ([3], [7]) when deciding what medium to communicate information; therefore, modeling the exact costs with using a particular tool for communication will allow a designer to compare them directly. We saw evidence of this tendency in the object list: despite a high cost of formulating utterances in the object list, groups universally used it, due to the reduction in group effort to maintain common ground thereafter.

Finally, the methods here are presented as methods by which an analyst can adapt and redesign a system off-line. However, improvements in live tagging of natural language discourse (as demonstrated by systems such as TRIPS-98 [6]) may allow automatic extraction and tracking of iotas, which would lead to a system that could continuously reassess use of information. By building continuously-updated models of user information use, the system could track live information use and adapt its interface dynamically to present the information in a more favorable or easy-to-use fashion.

\section{References}

1. Alterman, R., Feinman, A., Introne, J., Landsman, S.: Coordinating Representations in Computer-Mediated Joint Activities. Proceedings of 23rd Annual Conference of the Cognitive Science Society (2001)

2. Carpenter, T., Alterman, R.: A Taxonomy for Planned Reading. Proceedings of the 16th Annual Conference of the Cognitive Science Society (1994)

3. Clark, H.H., Brennan, S.E.: Grounding in communication. In Resnick, L.B., Levine, J., Teasley, S.D., editors, Perspectives on Socially Shared Cognition APA Press (1991)

4. DeCristofaro, J., Strube, M., McCoy, K.: Building a Tool for Annotating Reference in Discourse. Proceedings of the Workshop on the Relation of Discourse/Dialogue Structure and Reference, ACL'99 (1999)

5. Ellis, C.A., Gibbs, S.J., Rein, G.L.: Groupware: some issues and experiences. Communications of the ACM 34 (1991)

6. Ferguson, G., Allen, J.: TRIPS: An Intelligent Integrated Problem-Solving Assistant. Proceedings of the 15th National Conference on Artificial Intelligence (1998)

7. Gray, W., Fu, W.: Ignoring perfect knowledge in-the-world for imperfect knowledge in-the-head: implications of rational analysis for interface design. CHI Letters 3 (2001)

8. Hutchins, E.: Cognition in the Wild. Cambridge: MIT Press (1995)

9. Landsman, S., Alterman, R., Feinman, A., Introne, J.: VesselWorld and ADAPTIVE. Technical Report TR-01-213, Department of Computer Science, Brandeis University (2001). Presented as a demonstration at $C S C W-2000$ http://group.cs.brandeis.edu/group/papers/CSCWDemo.pdf

10. Sacks, H., Schegloff,, E., Jefferson, G.: A simplest systematics for the organisation of turn-taking for conversation. Language $\mathbf{5 0}$ (1974)

11. Seville, H., Ramsay, A.: Reference-based Discourse Structure for Reference Resolution. Proceedings of the Workshop on the Relation of Discourse/Dialogue Structure and Reference, ACL'99 (1999) 